\title{
A!
}

This is an electronic reprint of the original article.

This reprint may differ from the original in pagination and typographic detail.

Taskinen, Jani; Moilanen, Antti; Rekola, Heikki; Kuntze, Kim; Priimagi, Arri; Törmä, Päivi; Hakala, Tommi

\section{All-Optical Emission Control and Lasing in Plasmonic Lattices}

\section{Published in:}

ACS Photonics

DOI:

10.1021/acsphotonics.0c01099

Published: 21/10/2020

Document Version

Peer reviewed version

Please cite the original version:

Taskinen, J., Moilanen, A., Rekola, H., Kuntze, K., Priimagi, A., Törmä, P., \& Hakala, T. (2020). All-Optical Emission Control and Lasing in Plasmonic Lattices. ACS Photonics, 7(10), 28502858.

https://doi.org/10.1021/acsphotonics.0c01099

This material is protected by copyright and other intellectual property rights, and duplication or sale of all or part of any of the repository collections is not permitted, except that material may be duplicated by you for your research use or educational purposes in electronic or print form. You must obtain permission for any other use. Electronic or print copies may not be offered, whether for sale or otherwise to anyone who is not an authorised user. 


\title{
All-Optical Emission Control and Lasing in Plasmonic Lattices
}

\author{
Jani M. Taskinen, ${ }^{\dagger}$ Antti J. Moilanen, ${ }^{\dagger}$ Heikki Rekola ${ }^{\ddagger}$ Kim Kuntze, ${ }^{\top}$ Arri \\ Priimagi, "Päivi Törmä, ${ }^{\dagger}$ and Tommi K. Hakala*,‡ \\ $\dagger$ Department of Applied Physics, Aalto University, FI-00076 Aalto, Finland \\ $\ddagger$ Institute of Photonics, University of Eastern Finland, FI-80101 Joensuu, Finland \\ \Faculty of Engineering and Natural Sciences, Tampere University, FI-33101 Tampere, \\ Finland \\ E-mail: tommi.hakala@uef.fi
}

\begin{abstract}
We report on reversible all-optical emission control and lasing in plasmonic nanoparticle lattices. By incorporating photochromic molecules into the liquid gain medium composed of organic fluorescent molecules, we realize all-optical control over gain and absorption, the two key parameters associated with both conventional and nanoscale lasing. We demonstrate reversible photoswitching between two distinct modes of operation, 1) spontaneous emission to the lattice mode, characterized by broad emission linewidth, low emission intensity and large angular distribution and 2) lasing action, characterised by very narrow (sub-nm) linewidths due to emergence of increased gain and temporal coherence in the system, approximately three orders of magnitude increase in emission intensity, and narrow 0.7 degree angular divergence of the beam. A rate-equation model is employed to describe the operation of the switchable plasmonic laser. Our results provide the first demonstration of optically tunable losses
\end{abstract}


in plasmonic lattice lasers, which is an important milestone for the development of active plasmonics and paves the way for ultrafast all-optical switching of plasmonic nanolasers.

\section{Keywords}

Plasmonics, nanolasing, photoswitching, surface lattice resonance, optical control

\section{Introduction}

Metallic nanoparticles support surface plasmon resonances (SPRs) that exhibit strong nearfield character and tunability of the resonance condition via material choice, particle geometry, and refractive index of the surroundings. This makes SPRs a promising platform for nanoscale sensing applications. ${ }^{1,2}$ However, the high radiative and ohmic losses of SPRs have significantly limited the range of applicability of these resonances. In metallic nanoparticle arrays, the SPR of an individual particle can couple to other particles via radiation fields. The radiation-induced coupling of the lossy SPR mode with the low-loss diffracted order of the lattice induces hybrid modes known as surface lattice resonances (SLRs), with significantly reduced linewidths ${ }^{3-8}$ as compared to their uncoupled counterparts. Further, the tunability of the band structure via particle and lattice geometry provides a versatile platform for studying light-matter interactions at the nanoscale. ${ }^{9}$

Lasing action in IR and visible has been reported in nanoparticle arrays overlaid with optically pumped fluorescent molecules. Linewidth narrowing, increased temporal and spatial coherence, highly directional beam, and several orders of magnitude increase in emission intensity (for a given wavelength) was observed upon the onset of lasing. ${ }^{10,11}$ This together with the previous observations of lasing in plasmonic systems, ${ }^{12,13}$ provided an important milestone towards applications where plasmonic losses have typically been considered a hin- 
drance. Recently, plasmonic nanoparticle arrays have been shown to exhibit wavelengthtunable lasing when varying the refractive index of the gain medium, ${ }^{14}$ or the interparticle spacing on a stretchable substrate. ${ }^{15}$

Controlling lasing optically is desirable for the design of novel devices such as optical switches and memories. ${ }^{16}$ All-optical switching of lasing has been referred to modifying either the output intensity characteristics or the direction of lasing operation. ${ }^{17-19}$ In principle, tuning optical properties of lasing other than amplitude or directionality (such as phase, polarization, spatial/temporal distribution, etc.) could be beneficial for applications. In this work we focus on the on-off switching of lasing with photochromic molecules.

Photochromic molecules change their conformation reversibly upon exposure to light, modifying their absorption and emission spectra. ${ }^{20}$ Photochromic molecules have been utilized, for instance, in optical switches ${ }^{21,22}$ and transistors. ${ }^{23,24}$ They are also used for controlling the strength of light-matter interaction. ${ }^{25,26}$ Controlling optical gain by photochromic molecules was first proposed in a system consisting of photochromic molecules in a solid-state polymeric matrix. ${ }^{16}$ In the experiment, Pisignano et al. showed how amplified spontaneous emission can be switched on and off by all-optical control. Photoswitching of actual lasing was first reported in photonic crystals. ${ }^{27,28}$ Optical amplification at low temperatures ${ }^{29}$ and at room temperature ${ }^{30}$ has been demonstrated in optical microcavities. Very recently, even a single photon induced switching of lasing has been observed. ${ }^{31}$ Finally, a microcavity-based serial combination of all-optical components has been demonstrated, ${ }^{32}$ a necessary prerequisite for more complex logical operations. Plasmonic photoswitchable lasing has been theoretically proposed for multilayer plasmonic-photonic structures, ${ }^{33}$ and a photoswitchable spaser was experimentally demonstrated with gold nanoparticles in polymer shell covered with fluorescent proteins. ${ }^{34}$

Here, we demonstrate all-optical switching of lasing in plasmonic nanoparticle lattices using photochromic spiropyran derivatives. Unlike in Ref. ${ }^{34}$ where the wavelength of lasing was optically controlled, we demonstrate on-off switching of lasing in a plasmonic lattice. 
Our system is employing simple, commercially available compounds together with collective SLR modes instead of single particle plasmon resonances, allowing for a strong modulation of the beam divergence between the two regimes of operation. The high intensity modulation enabled by the system may be relevant for developing ultra-fast nanoscale optical circuits analogous to electronic binary circuits. ${ }^{35}$

Our sample consists of a square array of Ag nanoparticles (Fig. 1 (a)) overlaid with a liquid medium consisting of organic coumarin 6 (C6) molecules providing the optical gain, and photochromic indolinospiropyran molecules, which, depending on their conformational state, can be either strongly absorbing or nearly transparent at the lasing wavelength. The state can be reversibly changed by exposure to UV and visible light, see Fig. 1 (b), providing alloptical control of the absorption and emission properties of the hybrid system. A schematic of the sample is shown in Fig. 1 (c).

\section{Results}

When the particle periodicity $p$ in a plasmonic lattice equals the wavelength of radiation $\lambda$ in a medium with refractive index $n$, the radiation fields from all the particles interfere constructively at each particle location, creating a constant phase and increased dipole moment in each particle at $k=0$ at a wavelength $\lambda=p \times n$, i.e. at the $\Gamma$-point. This radiative coupling of individual nanoparticles can be used as a feedback mechanism for lasing and condensation. ${ }^{11,36-39}$

The absorbance of the photochromic molecule, indolinospiropyran (1',3'-dihydro-1',3',3'trimethyl-6-nitrospiro [2H-1-benzopyran-2,2'-(2H)-indole]), depends strongly on the conformational state of the molecule. In its relaxed closed form, spiropyran (SPI) is transparent

across the visible light spectrum, whereas the open form merocyanine (MC) absorbs strongly at around $2.28 \mathrm{eV}$. The switching from pure SPI to a photostationary state containing $c a$. 14\% MC (see Section S1 in the Supporting Information) is performed by UV light (365 nm 
a)

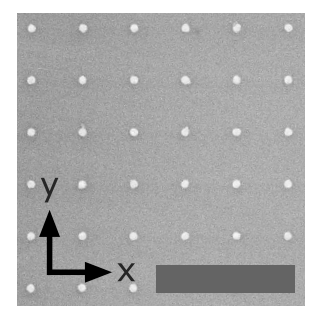

b)

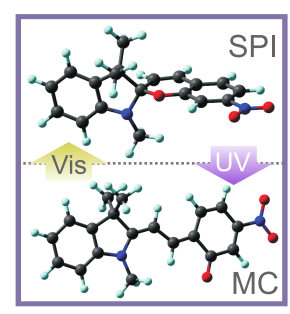

c)

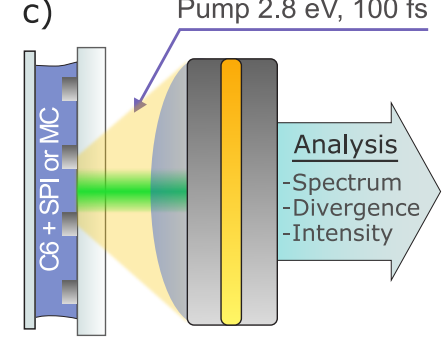

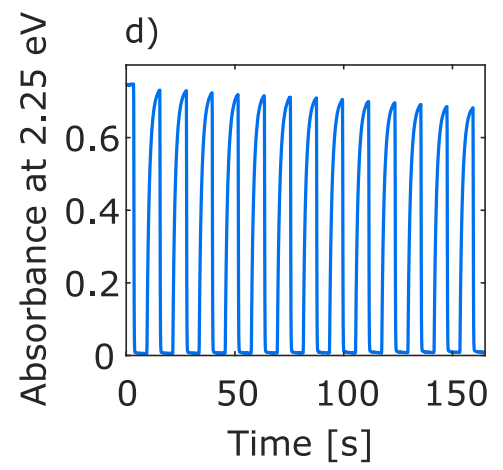

e)

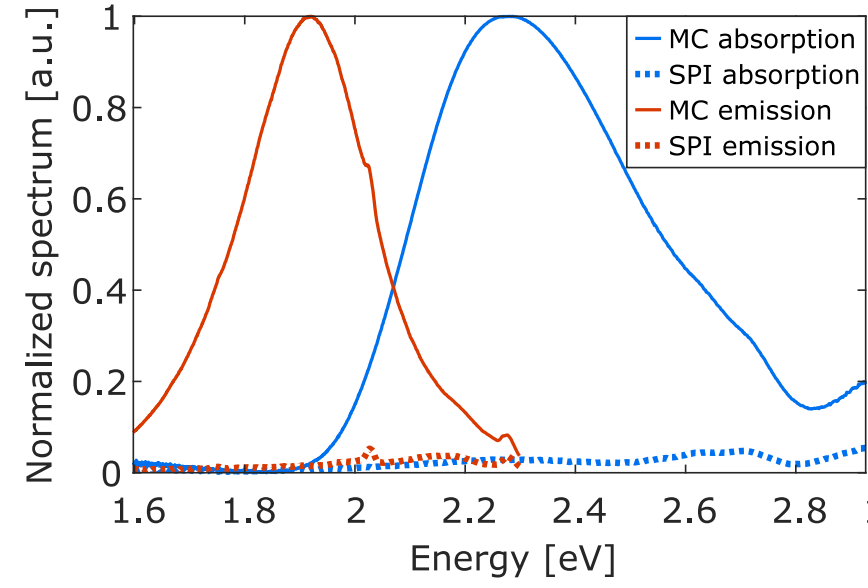

f)

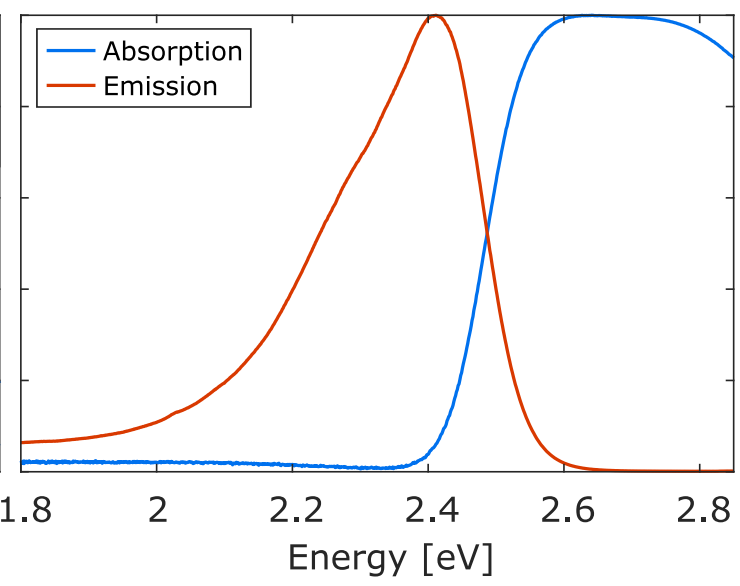

Figure 1: (a) A scanning electron micrograph of a typical sample, scale bar $1 \mu \mathrm{m}$. See Methods for description of the sample fabrication. (b) Illumination-induced isomerizations between SPI and MC. (c) For lasing measurements, a nanoparticle lattice overlaid with gain solution was pumped optically and the resulting emission was collected for angle and wavelength resolved data analysis. See Methods and Section S5 in the Supporting Information for details of the experimental setup. (d) Reversible absorbance of the photochromic molecules at $2.25 \mathrm{eV}$ during alternating UV and visible light treatments. See Methods for details of the spectrophotometric measurement. (e) The absorption (blue) and emission (red) of the pure SPI isomer (dashed) and the photostationary mixture of SPI and MC (solid). The used concentration in (d-e) was the same as in lasing measurements $(40 \mathrm{mM})$. (f) The absorption (blue) and emission (red) of C6 (30 mM). The used solvent in (d-f) was benzyl alcohol. 
wavelength, $50 \mathrm{~W} / \mathrm{m}^{2}$ ), and reversed by visible (green) light illumination. Fig. 1 (d) shows the reversible absorbance of the photochromic molecule during consecutive UV and visible light treatments. The MC-SPI isomerization also occurs via thermal relaxation but at room temperature it is significantly slower compared to using visible light illumination (see Section S2 in the Supporting Information). The normalized emission and absorption profiles of the SPI and MC forms are shown in Fig. 1 (e) which highlights the drastic difference between the two forms in terms of optical properties in the visible spectrum.

Fig. 1 (f) shows the normalized emission and absorption profiles of the used gain material C6, which was chosen as its emission profile overlaps with the absorption profile of MC. Additionally, the absorption profile of $\mathrm{C} 6$ allows it to be pumped at $450 \mathrm{~nm}(\approx 2.8 \mathrm{eV})$, which minimizes the effect of the pump laser on the form of the photochromic molecules as the absorbance of both SPI and MC is close to zero at this wavelength. Although the emission from C6 can cause the isomerization of some MC molecules back to the SPI form, the continuous replenishment of molecules from the dye bath prevents this from significantly changing the system operation in the timescale of our measurements. The plasmonic lattice optimized for lasing has a lattice periodicity of $350 \mathrm{~nm}$ which sets the $\Gamma$-point energy at $2.3 \mathrm{eV}$ with a refractive index of 1.54 in the fluorescent medium. The array size is $100 \times 100 \mathrm{\mu m}^{2}$, and the cylindrical nanoparticles have a diameter and height of $60 \mathrm{~nm}$ and $30 \mathrm{~nm}$, respectively.

The composite gain/loss medium consisting of C6 and photochromic molecules (in $30 \mathrm{mM}$ : $40 \mathrm{mM}$ ratio), was separately characterized, see Fig. 2. A small volume (7 pl) of the mixture was injected between a borosilicate microscope slide and a $22 \times 22 \mathrm{~mm}^{2}$ cover slip, and the emission was measured with several different UV exposure times, see Fig. 2 (a). In the absence of UV exposure, the emission resembles that of the C6 molecules (red dashed curve) with the maximum at around $2.41 \mathrm{eV}$. Increasing UV exposure gradually decreases the emission intensity, which is associated with the increased absorbance of the photochromic molecules. With sufficient (30 s) UV exposure (red solid curve), the emission reaches a minimum with approximately $20 \%$ of the initial emission intensity. Further, emission at $1.9 \mathrm{eV}$ 

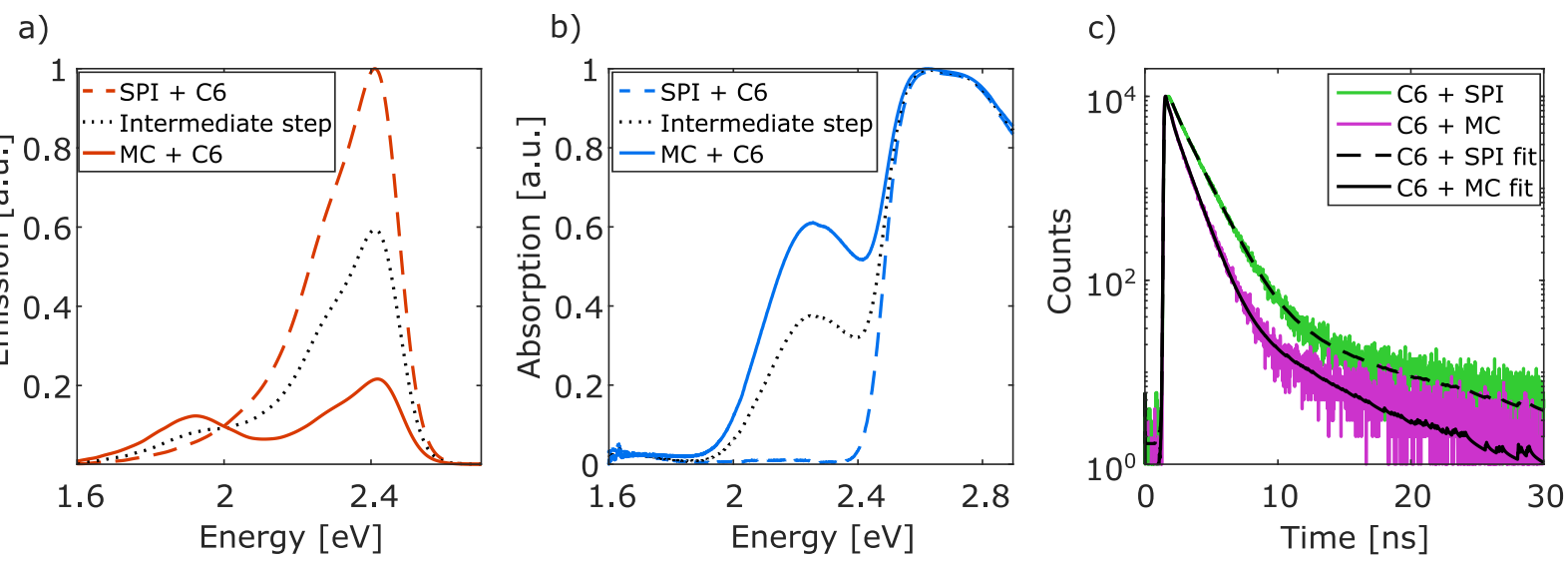

Figure 2: Emission and absorption properties of the composite gain medium. (a) Emission profile before (red dashed line) and after (red solid line) UV treatment. (b) Absorption before (blue dashed line) and after (blue solid line) UV treatment. The black dashed line in (a) and (b) shows an intermediate step between SPI and MC forms. (c) Lifetime measurements of the composite gain medium with the photochromic molecules in the SPI (green line) and MC (purple line) forms. Multiexponential fits to the lifetime data in SPI and MC forms are shown as dashed and solid black lines, respectively. The oscillations visible in the fitting curves above 20 ns are caused by background noise in the instrumental response function. See Methods for details of the time-resolved fluorescence spectroscopy setup.

is observed which we associate with the fluorescence of the $\mathrm{MC}$ form of the photochromic molecule. The absorption profile with similar configurations is shown in Fig. 2 (b). Before UV exposure (dashed blue line), the absorption resembles that of C6 but after sufficient UV treatment (blue solid line) the absorption peak of $\mathrm{MC}$ appears. In contrast to the measurement shown in Fig. 1 (d), here we use significantly longer exposure times for molecule switching as we wish to ensure maximal isomerization of the photochromic molecules. Due to the high concentration of both C6 and photochromic molecules in the solution, there is a possibility for non-radiative energy transfer between them. This was confirmed with timeresolved fluorescence spectroscopy: the fluorescence lifetime of C6 decreased from 1.42 to 1.06 ns upon SPI-MC isomerization (see Fig. 2 (c) and Section S3 in the Supporting Information). We note that the exact mechanism of the energy transfer requires further studies, and that both Förster- and Dexter-type mechanisms are plausible under these conditions.

For the lasing measurements, we overlay the nanoparticle array with the composite fluorescent solution and optically pump the structure with a femtosecond laser (450 nm center 
a)

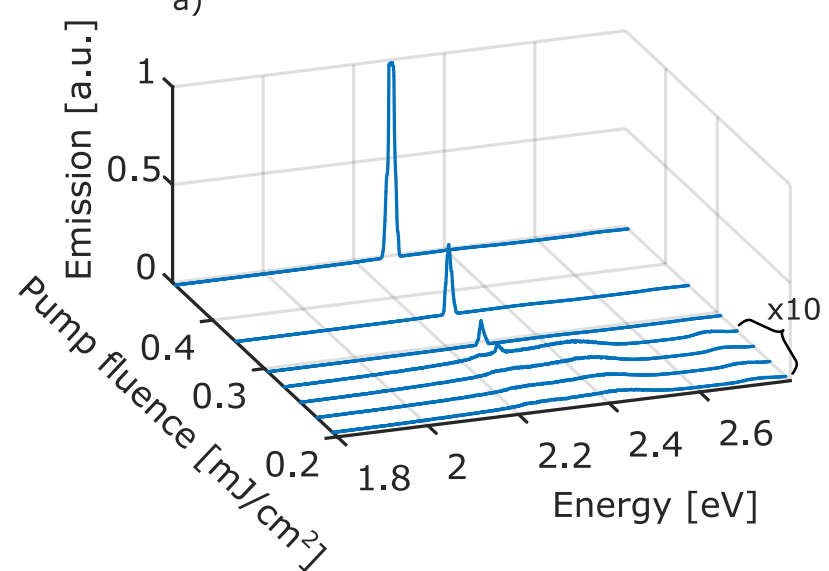

c)

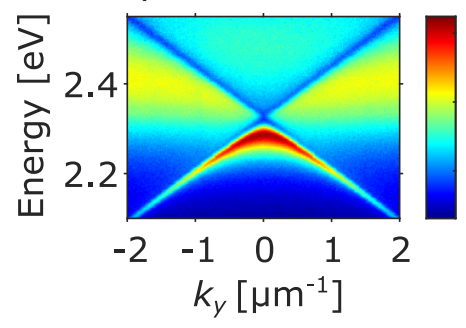

f)

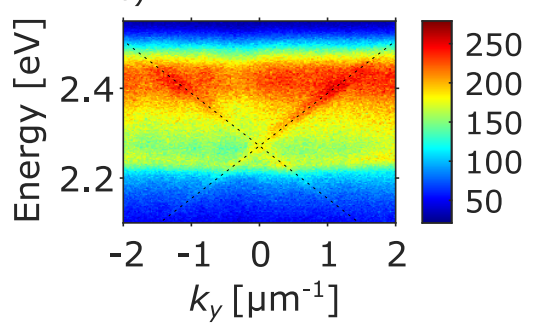

d)

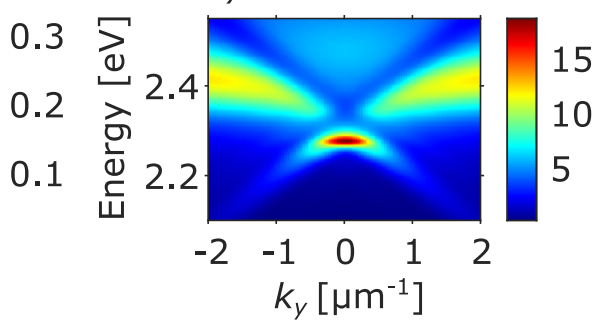

g)

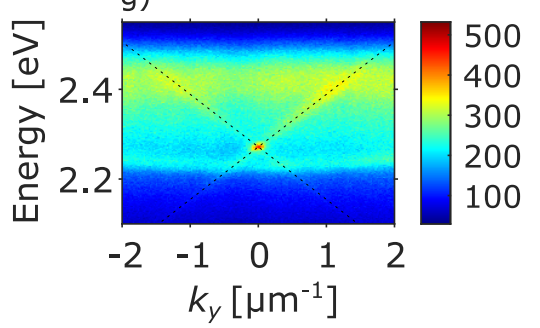

b)

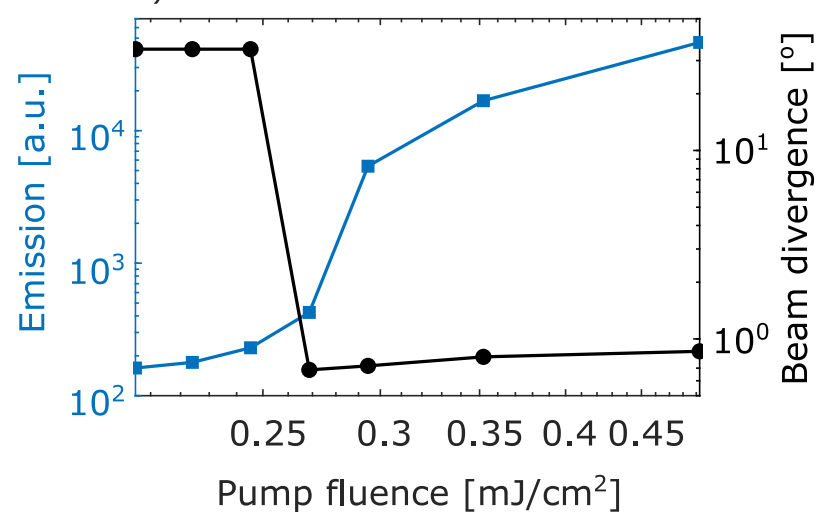

e)

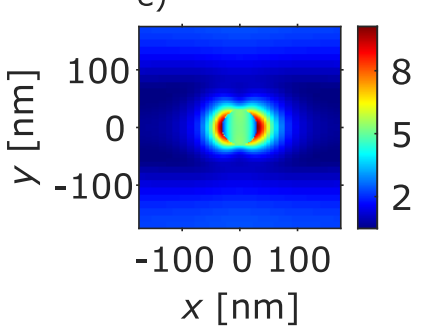

h)

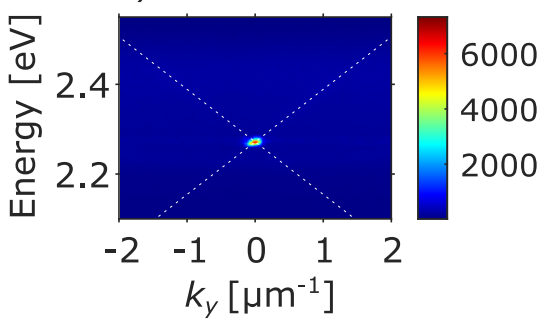

Figure 3: Emission below and above the lasing threshold. (a) Emission spectra as a function of energy for different pump fluences. (b) Integrated emission intensity (blue squares) near the $\Gamma$-point and beam divergence (black circles) at $k_{y}=0$ as a function of pump fluence. (c) Measured extinction of a typical bare array without fluorescent molecules calculated as $(1-T)$, where $T$ is transmission. (d) Simulated extinction efficiency of the array. The array period is $350 \mathrm{~nm}$ and the particle radius and height are $30 \mathrm{~nm}$. The simulation was done by using the coupled dipole approximation (CDA), ${ }^{40}$ see Methods. (e) The finite-difference time-domain (FDTD) simulation of a nanoparticle array illuminated with $x$-polarized plane wave at $2.3 \mathrm{eV}$. The color scale corresponds to the electric field profile $|E|$ normalized by the amplitude of the incoming plane wave. See Methods for details of the simulation. Also shown are the measured angle and energy resolved intensity data (f) below, (g) at and (h) above threshold pump fluence. 
wavelength, 100 fs pulse duration, $1 \mathrm{kHz}$ repetition rate). In Fig. 3 (a) the sample emission is shown as functions of energy and pump fluence, exhibiting a transition between two regimes: at low pump fluences, the emission intensity is low and the spectra are broad due to spontaneous emission. At higher pump fluences, though, the emission intensity increases in a nonlinear fashion, which is also evident from Fig. 3 (b). We note that this transition is accompanied with a rapid reduction in the emission beam divergence down to $0.7^{\circ}$. For the emission linewidths, see Section S6 in the Supporting Information.

Experimentally obtained and simulated dispersions of the array optimized for lasing are shown in Figs. 3 (c-d). The lattice dispersion shows the crossing of the $<0,+1>$ and $<0,-1>$ diffracted orders of the lattice at the $\Gamma$-point. Fig. 3 (e) shows the finite-difference time domain (FDTD) simulation of the nanoparticle array illuminated with a plane wave at the $\Gamma$-point energy. In Fig. 3 (f-h), we plot the angle and wavelength resolved emission data, revealing the crucial role of the nanoparticle lattice in both spontaneous emission and lasing regimes. The spontaneous emission angles are modified by the presence of the lattice, with the maximum emission taking place at the angles and energies that correspond to the SLR mode (dashed lines in Fig. 3 (f-h)). In the lasing regime, vast majority of photons are occupying a narrow energy $(\Delta E=11 \mathrm{meV})$ and wavevector $\left(\Delta k_{y}=0.137 \mathrm{~mm}^{-1}\right)$ range at the $\Gamma$-point of the nanoparticle lattice.

In order to simulate the composite system response, we use a rate equation model consisting of a 4-level system as shown in Fig. 4 (a). The model is defined with the following coupled rate equations: 


$$
\begin{aligned}
\frac{d n_{\mathrm{ph}}}{d t} & =\beta\left(n_{\mathrm{ph}}+1\right) \frac{N_{2}}{\tau_{21}}-\beta n_{\mathrm{ph}} \frac{N_{1}}{\tau_{21}}-\frac{n_{\mathrm{ph}}}{\tau_{\mathrm{ph}}} \\
\frac{d N_{0}}{d t} & =-P(t) N_{0}+\frac{N_{2}}{\tau_{20}}+\frac{N_{1}}{\tau_{10}} \\
\frac{d N_{1}}{d t} & =\beta n_{\mathrm{ph}} \frac{\left(N_{2}-N_{1}\right)}{\tau_{21}}+\frac{N_{2}}{\tau_{21}}-\frac{N_{1}}{\tau_{10}} \\
\frac{d N_{2}}{d t} & =-\beta n_{\mathrm{ph}} \frac{\left(N_{2}-N_{1}\right)}{\tau_{21}}-\frac{N_{2}}{\tau_{21}}-\frac{N_{2}}{\tau_{20}}+\frac{N_{3}}{\tau_{32}} \\
\frac{d N_{3}}{d t} & =P(t) N_{0}-\frac{N_{3}}{\tau_{32}},
\end{aligned}
$$

where the populations of each level are denoted with $N_{i}$ and the lifetimes of transitions between levels $i-j$ with $\tau_{i j}$. Here, $n_{\mathrm{ph}}$ is the photon number in the lasing mode. Pump rate $P(t)$ is proportional to the pump intensity that has a Gaussian temporal profile centered at $150 \mathrm{fs}$ and full width at half maximum of $100 \mathrm{fs}$. The lifetime of the SLR mode $\tau_{\mathrm{ph}}=$ $85 \mathrm{fs}$ is obtained from the dispersion linewidth at $k_{y}=0$ in Fig. 3 (c). The spontaneous emission factor $\beta$ is given by the ratio between spontaneously emitted photons and all photons emitted into the lasing mode. ${ }^{41,42}$ Increase of total intensity of about 2 orders of magnitude in Fig. 3 (b) corresponds to $\beta=0.01$.

The 2-1 transition of the 4-level system is resonant with the cavity mode and therefore corresponds to the radiative spontaneous emission lifetime of C6 $\left(\tau_{21}\right)$. The 2-0 transition is non-resonant and relates to a non-radiative lifetime $\left(\tau_{20}\right)$. Intrinsic non-radiative decay of C6 is on the order of $11.8 \mathrm{~ns}^{43}$ which is significantly longer than any other time scale in the system dynamics. Therefore, we neglect the effect of the intrinsic non-radiative decay and use the parameter $\tau_{20}$ to model the effect of additional non-radiative energy transfer taking place upon SPI-MC isomerization. We obtain the lifetimes $\tau_{21}=1.42 \mathrm{~ns}$ and $\tau_{20}=4.2 \mathrm{~ns}$ from the fluorescence lifetime measurements (see Section S4 in the Supporting Information). As 
a)

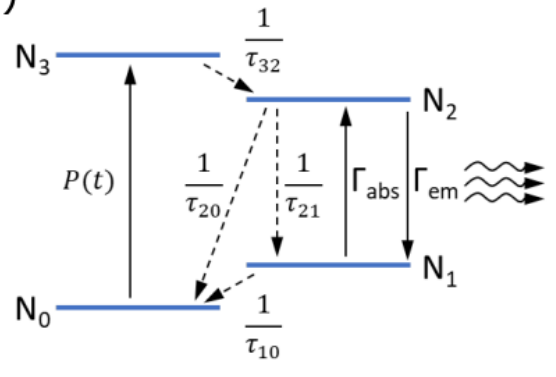

c)

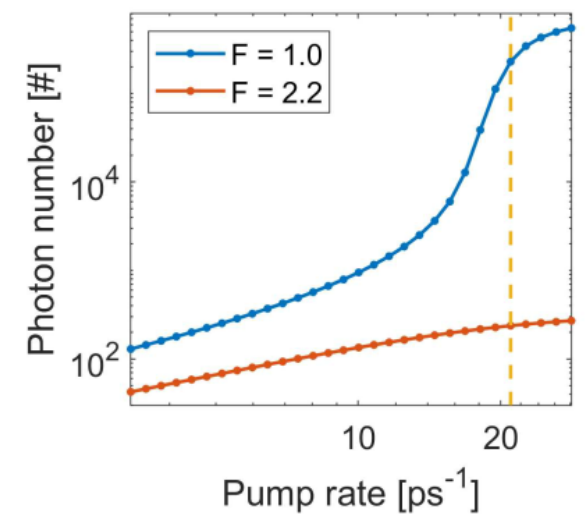

b)

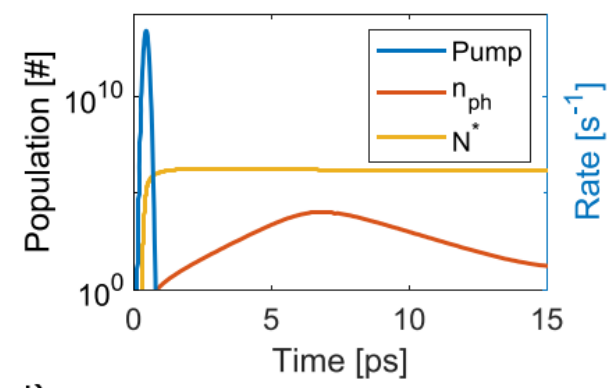

d)

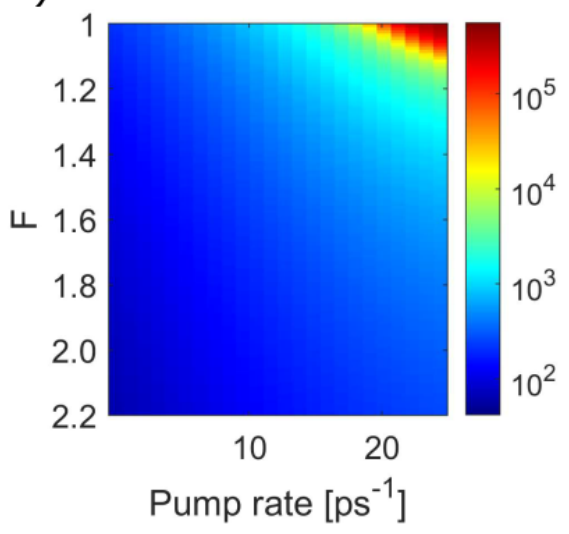

Figure 4: Rate equation simulation of photoswitching. (a) Schematic of the 4-level rate equation model. (b) Rate equation simulation showing the pump pulse (blue), population inversion $N^{*}=N_{2}-N_{1}$ (yellow), and output lasing pulse $n_{\mathrm{ph}}$ (red solid line) as a function of time. The result is computed just above the lasing threshold at pump rate $21 \mathrm{ps}^{-1}$ (yellow dashed line). The full width at half maximum of the output lasing pulse is $2.6 \mathrm{ps}$. (c) Photon number of the cavity mode, $n_{\mathrm{ph}}$, as a function of pump rate for $F=1.0$ and $F=2.2$. (d) Photon number of the cavity mode, $n_{\mathrm{ph}}$, as a function of pump rate and $F$. 
vibrational relaxation times for coumarin dyes are typically on the order of $100 \mathrm{fs} . .1 \mathrm{ps},{ }^{44,45}$ we set $\tau_{32}$ and $\tau_{10}$ to $0.5 \mathrm{ps}$. For the simulations we use $2 \times 10^{6}$ molecules and let the simulation run for a sufficiently long time so that all excitations created by the pump have decayed (20 ns).

To qualitatively model the effect of photoswitching, we modify the loss rate of the photon mode by a factor $F$, namely as $F \times n_{\mathrm{ph}} / \tau_{p h}$ in Eq. (1). The factor $F$ therefore accounts for the overall increase of photon loss from the lasing mode due to increased absorption imposed by switching the photochromic molecules from the SPI to the MC form. We map the factor $F$ to experiments by reproducing the measured spontaneous emission intensities for both forms of the dye near the $\Gamma$-point. Switching the molecules to the absorbing form reduces the spontaneous emission intensity to approximately $30 \%$ of the initial value. In the simulation this is reproduced by increasing the loss factor $F$ from 1.0 to 2.2 . Fig. 4 (c) shows the pump dependence curves for $F=1.0$ (SPI form of the molecule) and $F=2.2$ (MC form of the molecule). In the spontaneous emission regime the ratio of photon numbers between $F=2.2$ and $F=1.0$ curves is around $30 \%$. In the case of $F=1.0$ the model reproduces the experimentally obtained lasing threshold curve. For $F=2.2$, no onset of lasing is observed in the range of pump fluences studied. At pump rate $21 \mathrm{ps}^{-1}$ (yellow dashed line), the ratio of output intensities between the two forms of the molecule is $10^{3}$.

Fig. $4(\mathrm{~d})$ presents the photon number as a function of pump rate and $F$. The simulation suggests that already a 20-30\% increase in photon loss rate would enable 1-2 orders of magnitude modulation of the lasing signal. Simulations in the time domain, as exemplified by Fig. 4 (b), show that the lasing pulse comes out on a few picosecond timescale. This is in accordance with the previous studies in plasmonic lattices demonstrating ultrafast lasing pulse generation. ${ }^{41}$

Finally, in Figs. 5 (a-b) we measure the angle and energy resolved emission spectra at a fixed pump fluence of $0.19 \mathrm{mJcm}^{-2}\left(\sim 1.1 \mathrm{P}_{\mathrm{th}}\right)$ after exposing the sample to (a) visible and (b) UV light (30 s exposure time). In Fig. 5 (a), the system exhibits lasing similar to 
that observed in Fig. 3. After the UV exposure, however, the sample transits to spontaneous emission regime due to increased overall losses in the mode volume of the plasmonic nanoparticle lattice. Remarkably, by visible light exposure, the lasing action can be recovered. Figs. 5 (c-e) show the emission intensity at $\Gamma$-point $\left(k_{y}=0\right)$ as well as the linewidths and beam divergences for alternating UV and visible light exposures, demonstrating the repeatibility of all-optical control of lasing in nanoparticle lattices. The switching is established over 2-3 orders of magnitude, which is in agreement with the rate equation simulation. We also observe approximately 100 and 50 fold modulation in linewidth and beam divergence, respectively.

a)

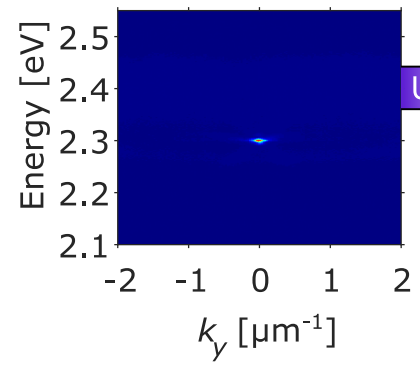

c)

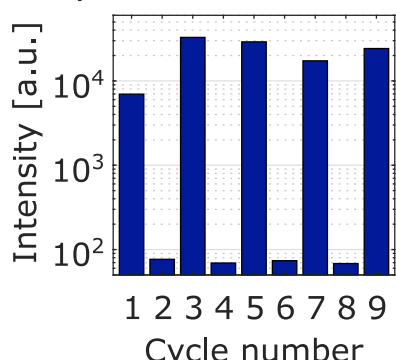

d)

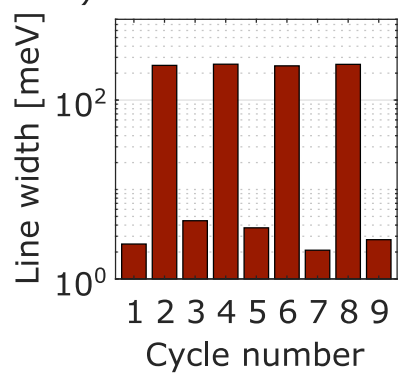

b)

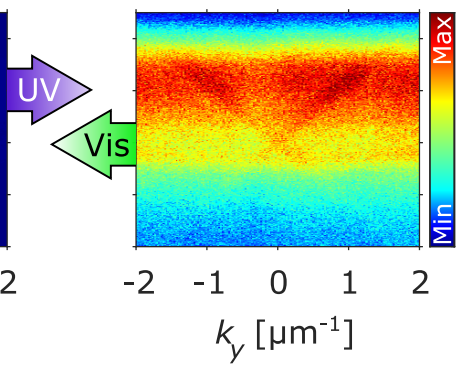

e)

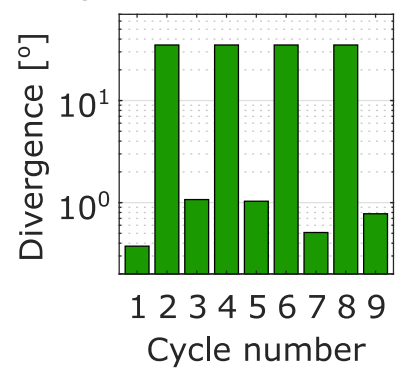

Figure 5: Optical control of lasing. (a-b) Normalized emission intensity of a photoswitchable lasing sample after (a) visible and (b) UV light illumination. (c) Integrated intensity values near the $\Gamma$-point, (d) measured emission linewidths (full width at half maximum) at $k_{y}=0$ and (e) beam divergences after alternating UV and visible light exposures.

As concluded from comparing the sample emission for both forms of the photochromic dye, the overall increase in the photon loss rate causes the spontaneous emission intensity to drop to about $30 \%$ of the initial value when the photochromic molecules are switched to the $\mathrm{MC}$ form. Importantly, due to the highly nonlinear response of plasmonic lasers, 
the additional losses caused by the SPI-MC switching are sufficient to produce orders of magnitude variation in the output intensity.

The results of the time domain simulations suggest that with the realistic parameters used in the model, the lasing dynamics occur on a picosecond timescale, in agreement with previous findings. ${ }^{41,46}$ Combining our system with photochromic compounds capable of switching their conformation on a 1-10 ps timescale (for instance, diarythelene molecules ${ }^{47}$ ) could provide ultrafast, all-optical switching with exciting future prospects toward optoelectronic switches and memories that could operate at up to THz-frequencies.

We point out that the functional components of the presented system span over particularly wide range of different length scales (6 orders of magnitude). First, the composite gain medium is a mixture of 2 different molecules of approximately $1 \mathrm{~nm}$ size. Second, their (UV and visible light tunable) intermolecule (donor to acceptor) distances range from infinite to approximately $3 \mathrm{~nm}$. Third, the plasmonic nanoparticle resonances have high near field enhancements within the range of $10 \mathrm{~nm}$ from the surface of the particle. Fourth, plasmonic nanoparticle lattices exhibit resonances having coherence lengths on the order of $10 \mu \mathrm{m}$ due to radiative coupling between individual nanoparticles, and, finally, the lasing in such lattices exhibits coherence lengths in excess of $1 \mathrm{~mm}$, which appears to be only limited by the lattice size or the pump area. ${ }^{46}$ Very recently, the challenges of analysing light-matter interactions and energy transfer in such multiscale systems have been recognised and addressed by an elaborate study which combines classical (macroscopic) and quantum mechanical (microscopic) descriptions. ${ }^{48}$ To understand the potential role of plasmon-enhanced energy transfer in our results, such mesoscopic approach should be combined with description of the nonlinear dynamics caused by gain and losses. We view this challenge as a fertile ground for novel findings and a well justified motivation for further studies in such tunable multiscale systems. 


\section{Conclusions}

To summarize, we have shown how photochromic molecules enable all-optical emission control and switching of lasing in nanoparticle arrays. By combining the organic dye with photochromic molecules we added a reversible, optically controllable loss mechanism to the system. Combined with the non-linear response of the plasmonic laser, it enables reversible switching between spontaneous emission and lasing regimes, with 2-3 orders of magnitude modulation in light output intensity. Our observations are qualitatively explained by a 4-level rate equation model. In addition to the strong intensity modulation, we observe 100 and 50 fold modulation in linewidth and beam divergence, respectively, when switching between the two regimes. While optical gain and lasing have been extensively investigated in plasmonic systems, the control over the losses has been scarcely studied. All-optical, reversible control provided by photochromic molecules over this quantity is particularly appealing as it opens the route towards actively tunable plasmonics, in time-scales limited only by the switching time of the molecules which can reach the $\mathrm{THz}$ scale.

\section{Methods}

\section{Sample fabrication}

Square arrays of silver nanoparticles were fabricated on borosilicate glass slides using standard electron beam lithography methods. The substrates were spin-coated with a polymethyl methacrylate (PMMA A4) resist layer which was solidified on a hot plate. The PMMA layer was patterned by exposing it to an electron beam and developed in 1:3 methyl isobutyl ketone : isopropanol solution. The nanoparticle diameter was $60 \mathrm{~nm}$, the array size $100 \times 100 \mu^{2}$ and the array periodicity $350 \mathrm{~nm}$. A $2 \mathrm{~nm}$ titanium adhesion layer and a $30 \mathrm{~nm}$ silver layer were evaporated on the patterned PMMA layer, followed by lift-off. For transmission (lasing)

measurements the nanoparticle arrays were immersed in index-matching oil (fluorescent gain 
medium) and covered with a microscope cover slip.

\section{Angle-resolved intensity spectroscopy}

Angle-resolved intensity measurements were obtained by collecting light emitted by or transmitted through the sample with an objective (10x, $0.3 \mathrm{NA})$ whose back focal plane was focused to the entrance slit of a spectrometer. The angle of light exiting the sample $\theta_{y}$ is

mapped on the spectrometer slit as $k_{y}=k_{0} \sin \theta_{y}=2 \pi / \lambda_{0} \sin \theta_{y}$ where $\lambda_{0}$ is the free space wavelength. Each point on the slit and likewise each pixel row on the charge-coupled device camera corresponds to a $k_{y}$ value while the pixel columns are used to resolve the energy spectrum at each $k_{y}$ such that $E=h c / \lambda_{0}$ where $h$ is the Planck constant and $c$ is the speed of light. An iris was used in the real image plane of the setup to filter out any light emerging from outside the array. In the lasing measurements the sample was pumped with femtosecond laser pulses $\left(1 \mathrm{kHz}\right.$ repetition rate, $100 \mathrm{fs}$ pulse duration, $450 \mathrm{~nm}$ center wavelength, $45^{\circ}$ incident angle) with a flat pulse profile.

\section{Time-resolved fluorescence spectroscopy}

Fluorescence intensity decay curves were measured by using a time-correlated single photon counting (TCSPC) system (PicoQuant, GmBH) equipped with PicoHarp 300 controller and a PDL 800-B driver. LDH-P-C-483 laser head was used to excite the sample at $483 \mathrm{~nm}$ with a time resolution of $\sim 110 \mathrm{ps}$. The fluorescence signal was detected by means of microchannel plate photomultiplier tube (Hamamatsu R2809U). Cutoff filter of transmission > $520 \mathrm{~nm}$ was applied to reduce the impact of the excitation light scattering. Fluorescence decays were monitored at $540 \mathrm{~nm}$ for all samples to maximize the signal. Instrumental response function was measured separately at monitoring wavelength $483 \mathrm{~nm}$ and used for deconvolution 
analysis of the fluorescence decays followed by their fitting by sum of exponents

$$
I(t, \lambda)=\sum_{i} a_{i} e^{-t / \tau_{i}}
$$

where $\tau_{i}$ is the fluorescence lifetime and $a_{i}$ is the pre-exponential factor.

\section{Spectrophotometric measurements}

Spectrophotometric studies were performed with an Agilent Cary 60 spectrophotometer and a large custom-built cavity with an Ocean Optics qpod 2e temperature-controlled cuvette holder and a Prior Scientific Lumen-1600 light source. The light source includes LEDs of different wavelengths and the wavelengths used for switching the photochromic molecules were $365 \mathrm{~nm}$ (SPI to MC) and $550 \mathrm{~nm}(\mathrm{MC}$ to SPI).

\section{Simulations}

The FDTD simulations were conducted using Lumerical's FDTD Solutions package. The nanoparticle was modeled as a $60 \mathrm{~nm}$ diameter, $30 \mathrm{~nm}$ tall silver cylinder in the middle

of the simulation volume using tabulated values for the permittivity. ${ }^{49}$ The particle was surrounded by a $1 \mathrm{~nm}$ shell of a dielectric material with a refractive index of $1.54+0.2 i$, while the background refractive index of the simulation was set to 1.54 . The lossy shell was used to reduce meshing related artefacts at the silver boundary in the field profile. The particle was meshed using a $0.5 \mathrm{~nm}$ regular mesh, and the boundary conditions were antisymmetric for $x$, symmetric for $y$, and perfectly matched layers for $z$, leaving only one quarter of the nanoparticle in the final simulation volume. The period in $x$ and $y$ was $350 \mathrm{~nm}$, and the simulation height in $z 2 \mu \mathrm{m}$. An $x$-polarized plane wave traveling along the $z$-axis was used excite the system, and the field profile was recorded in the $x y$-plane at $z=0$.

We use coupled dipole approximation (CDA) to compute the extinction spectrum of a two-dimensional periodic array of nanoparticles. See Ref. ${ }^{40}$ and references therein for a 
detailed description of the method. In CDA, each nanoparticle in the array is treated as a single electric dipole characterized by its induced polarization

$$
\vec{P}_{n}=\alpha\left(\vec{E}_{n}^{\mathrm{inc}}+\vec{E}_{n}^{\mathrm{sc}}\right),
$$

where $\vec{E}_{n}^{\mathrm{inc}}$ is the incident field acting on the $n$th particle, and $\vec{E}_{n}^{\mathrm{sc}}$ is the field resulting from scattering by all the other nanoparticles. The polarizability $\alpha$ is calculated for an ellipsoid nanoparticle under the modified long-wavelength approximation. ${ }^{40,50}$ The scattered field can be written as

$$
\vec{E}_{n}^{\mathrm{sc}}=\sum_{m=1, m \neq n}^{N} \underline{\vec{G}}\left(\vec{r}_{n}, \vec{r}_{m}\right) \vec{P}_{m}
$$

where $\underline{\vec{G}}$ is the electric-field dyadic Green's function $\left(R=|\vec{R}|=\left|\vec{r}_{n}-\vec{r}_{m}\right|\right)$

$$
\underline{\vec{G}}\left(\vec{r}, \vec{r}^{\prime}\right)=\frac{e^{i k R}}{4 \pi \epsilon_{0} R}\left[(\underline{\vec{I}}-\overrightarrow{\hat{R}} \overrightarrow{\hat{R}})+\frac{i k R-1}{k^{2} R^{2}}(\underline{\vec{I}}-3 \overrightarrow{\hat{R}} \overrightarrow{\hat{R}})\right]
$$

The Green's function in Eq. (9) is singular at $\vec{r}_{n}=\vec{r}_{m}$. In the dipole approximation, it is conventional to regularize the Green's function by excluding the self-terms representing the interaction of the nanoparticle with itself. ${ }^{51}$ Substituting the scattered field in Eq. (8) into Eq. (7) leads to a linear system

$$
\sum_{m=1}^{N} \vec{A}_{n m} \vec{P}_{m}=\vec{E}_{n}^{\mathrm{inc}}
$$

where the elements of the interaction matrix $\underline{\vec{A}}$ are given by

$$
\underline{\vec{A}}_{n m}= \begin{cases}\underline{\underline{\alpha}}^{-1} \underline{\underline{I}}, & n=m, \\ -\underline{\vec{G}}\left(\vec{r}_{n}, \vec{r}_{m}\right), & n \neq m .\end{cases}
$$

Note that whilst the self-terms of the Green's function are neglected, the interaction matrix $\underline{\vec{A}}$ contains the single-nanoparticle polarizabilities in the diagonal, representing the selfinteraction of the nanoparticles. The induced polarization of the nanoparticles, obtained by 
solving the linear system Eq. (10), are used to calculate the extinction cross section: ${ }^{52}$

$$
C_{\text {ext }}=\frac{4 \pi k}{\left|E^{\text {inc }}\right|^{2}} \sum_{n=1}^{N} \operatorname{Im}\left(\vec{E}_{n}^{\text {inc* }} \cdot \vec{P}_{n}\right) .
$$

Extinction efficiency is obtained from the cross section as $Q_{\text {ext }}=C_{\text {ext }} / A$, where $A$ is the surface area of the nanoparticle array. The results shown in Fig. 3 (d) were obtained for $30 \times 30$ array of nanoparticles excited with a plane wave polarized in the $x$-direction. The simulation parameters were as in the experiments: periodicity was $350 \mathrm{~nm}$ and the particle radius and height were $30 \mathrm{~nm}$. Refractive index of the background was 1.52 . We used tabulated values for the permittivity of silver. ${ }^{49}$ 


\section{Acknowledgments}

Funding: This work is part of the Academy of Finland Flagship Programme, Photonics Research and Innovation (PREIN), decisions 320165, 320166 and 320167. This work was supported by the Academy of Finland under project numbers 322002, 303351, 307419, 327293, 324353, 318987 (QuantERA project RouTe), and 318937 (PROFI), by Centre for Quantum Engineering (CQE) at Aalto University, and by the European Research Council (Starting Grant Project PHOTOTUNE, decision number 679646). Part of the research was

performed at the Micronova Nanofabrication Centre, supported by Aalto University. A.J.M acknowledges financial support by the Jenny and Antti Wihuri Foundation. Author contributions: T.K.H., P.T., and A.P. initiated and supervised the research. J.M.T. fabricated the samples. J.M.T., H.R., T.K.H., and K.K. conducted the experiments. J.M.T., T.K.H., A.J.M., and K.K. did the data analysis. A.J.M. performed the CDA and rate equation, and H.R. the FDTD simulations. T.K.H., A.J.M., and J.M.T. wrote the manuscript with input from all authors. All authors discussed the results and implications. Competing interests: The authors declare no competing financial interests.

\section{Supporting Information}

The Supporting Information is available free of charge at http://pubs.acs.org.

Photostationary state composition; Thermal lifetime of the MC form; Fluorescence lifetimes; Lifetimes in the rate-equation model; Schematic of the angle-resolved measurement setup; Sample emission linewidth as a function of pump fluence

\section{References}

(1) Klar, T. A. In Nanophotonics with Surface Plasmons; Shalaev, V. M., Kawata, S., Eds.; Advances in Nano-Optics and Nano-Photonics; Elsevier: Amsterdam, 2007; pp 219 - 
270.

(2) Homola, J.; Piliarik, M. In Surface Plasmon Resonance Based Sensors; Homola, J., Ed.; Springer Berlin Heidelberg: Berlin, Heidelberg, 2006; pp 45-67.

(3) Zou, S.; Janel, N.; Schatz, G. C. Silver nanoparticle array structures that produce remarkably narrow plasmon lineshapes. J. Chem. Phys. 2004, 120, 10871-10875.

(4) García de Abajo, F. J. Colloquium: Light scattering by particle and hole arrays. Rev. Mod. Phys. 2007, 79, 1267-1290.

(5) Kravets, V. G.; Schedin, F.; Grigorenko, A. N. Extremely Narrow Plasmon Resonances Based on Diffraction Coupling of Localized Plasmons in Arrays of Metallic Nanoparticles. Phys. Rev. Lett. 2008, 101, 087403.

(6) Auguié, B.; Barnes, W. L. Collective Resonances in Gold Nanoparticle Arrays. Phys. Rev. Lett. 2008, 101, 143902.

(7) Rodriguez, S. R. K.; Abass, A.; Maes, B.; Janssen, O. T. A.; Vecchi, G.; Gómez Rivas, J. Coupling Bright and Dark Plasmonic Lattice Resonances. Phys. Rev. X 2011, 1, 021019 .

(8) Wang, W.; Ramezani, M.; Väkeväinen, A.; Törmä, P.; Rivas, J.; Odom, T. The Rich Photonic World of Plasmonic Nanoparticle Arrays. Mater. Today 2018, 21, 303.

(9) Törmä, P.; Barnes, W. L. Strong Coupling between Surface Plasmon Polaritons and Emitters: A Review. Rep. Prog. Phys. 2015, 78, 013901.

(10) Zhou, W.; Dridi, M.; Suh, J. Y.; Kim, C. H.; Co, D. T.; Wasielewski, M. R.; Schatz, G. C.; Odom, T. W. Lasing action in strongly coupled plasmonic nanocavity arrays. Nat. Nanotechnol. 2013, 8, 506-511. 
(11) Hakala, T. K.; Rekola, H. T.; Väkeväinen, A. I.; Martikainen, J.-P.; Nečada, M.; Moilanen, A. J.; Törmä, P. Lasing in Dark and Bright Modes of a Finite-Sized Plasmonic Lattice. Nat. Commun. 2017, 8, 13687.

(12) Hill, M.; Gather, M. Advances in small lasers. Nat. Photonics 2014, 8, 908--918.

(13) Wang, D.; Wang, W.; Knudson, M. P.; Schatz, G. C.; Odom, T. W. Structural Engineering in Plasmon Nanolasers. Chemical Reviews 2018, 118, 2865-2881.

(14) Yang, A.; Hoang, T. B.; Dridi, M.; Deeb, C.; Mikkelsen, M. H.; Schatz, G. C.; Odom, T. W. Real-time tunable lasing from plasmonic nanocavity arrays. Nat. Commun. 2015, 6, 6939 .

(15) Wang, D.; Bourgeois, M. R.; Lee, W.-K.; Li, R.; Trivedi, D.; Knudson, M. P.; Wang, W.; Schatz, G. C.; Odom, T. W. Stretchable Nanolasing from Hybrid Quadrupole Plasmons. Nano Lett. 2018, 18, 4549-4555.

(16) Pisignano, D.; Mele, E.; Persano, L.; Athanassiou, A.; Fotakis, C.; Cingolani, R. Optical Gain from the Open Form of a Photochromic Molecule in the Solid State. J. Phys. Chem. B 2006, 110, 4506-4509.

(17) Zervos, C.; Frogley, M. D.; Phillips, C. C.; Kundys, D. O.; Wilson, L. R.; Hopkinson, M.; Skolnick, M. S. All-Optical Switching in Quantum Cascade Lasers. Appl. Phys. Lett. 2007, 90, 053505 .

(18) Troppenz, U.; Hamacher, M.; Radziunas, M.; Heidrich, H. Optical switching of clockwise/anti-clockwise lasing in bus coupled microrings. International Conference on Indium Phosphide and Related Materials, 2005. 2005; pp 307-310.

(19) Li, B.; Memon, M. I.; Mezosi, G.; Yuan, G.; Wang, Z.; Sorel, M.; Yu, S. All-Optical Response of Semiconductor Ring Laser to Dual-Optical Injections. IEEE Photonics Technol. Lett. 2008, 20, 770-772. 
(20) Nakatani, K.; Piard, J.; Yu, P.; Métivier, R. Photochromic Materials; John Wiley \& Sons, Ltd, 2016; pp 1-45.

(21) Pala, R. A.; Shimizu, K. T.; Melosh, N. A.; Brongersma, M. L. A Nonvolatile Plasmonic Switch Employing Photochromic Molecules. Nano Lett. 2008, 8, 1506-1510.

(22) Zheng, Y. B.; Kiraly, B.; Cheunkar, S.; Huang, T. J.; Weiss, P. S. Incident-AngleModulated Molecular Plasmonic Switches: A Case of Weak Exciton-Plasmon Coupling. Nano Lett. 2011, 11, 2061-2065.

(23) Börjesson, K.; Herder, M.; Grubert, L.; Duong, D. T.; Salleo, A.; Hecht, S.; Orgiu, E.; Samorì, P. Optically Switchable Transistors Comprising a Hybrid Photochromic Molecule/n-Type Organic Active Layer. J. Mater. Chem. C 2015, 3, 41564161.

(24) Hou, L.; Zhang, X.; Cotella, G. F.; Carnicella, G.; Herder, M.; Schmidt, B. M.; Pätzel, M.; Hecht, S.; Cacialli, F.; Samorì, P. Optically Switchable Organic LightEmitting Transistors. Nat. Nanotechnol. 2019, 14, 347-353.

(25) Schwartz, T.; Hutchison, J. A.; Genet, C.; Ebbesen, T. W. Reversible Switching of Ultrastrong Light-Molecule Coupling. Phys. Rev. Lett. 2011, 106, 196405.

(26) Moilanen, A. J.; Hakala, T. K.; Törmä, P. Active Control of Surface Plasmon-Emitter Strong Coupling. ACS Photonics 2018, 5, 54-64.

(27) Furumi, S.; Fudouzi, H.; Sawada, T. Dynamic Photoswitching of Micropatterned Lasing in Colloidal Crystals by the Photochromic Reaction. J. Mater. Chem. 2012, 22, 2151921528.

(28) Sridharan, D.; Bose, R.; Kim, H.; Solomon, G. S.; Waks, E. A reversibly tunable photonic crystal nanocavity laser using photochromic thin film. Opt. Express 2011, 19, $5551-5558$. 
(29) Savvidis, P. G.; Baumberg, J. J.; Stevenson, R. M.; Skolnick, M. S.; Whittaker, D. M.; Roberts, J. S. Angle-Resonant Stimulated Polariton Amplifier. Phys. Rev. Lett. 2000, $84,1547-1550$.

(30) Zasedatelev, A. V.; Baranikov, A. V.; Urbonas, D.; Scafirimuto, F.; Scherf, U.; Stöferle, T.; Mahrt, R. F.; Lagoudakis, P. G. A room-temperature organic polariton transistor. Nat. Photonics 2019, 13, 378-383.

(31) Zasedatelev, A.; Baranikov, A. V.; Sannikov, D.; Urbonas, D.; Scafirimuto, F.; Shishkov, V. Y.; Andrianov, E. S.; Lozovik, Y. E.; Scherf, U.; Stöferle, T.; Mahrt, R. F.; Lagoudakis, P. G. Organic single-photon switch. arXiv 2020, 2005.05811.

(32) Baranikov, A. V.; Zasedatelev, A. V.; Urbonas, D.; Scafirimuto, F.; Scherf, U.; Stöferle, T.; Mahrt, R. F.; Lagoudakis, P. G. All-optical cascadable universal logic gate with sub-picosecond operation. arXiv 2020, 2005.04802.

(33) Scotognella, F. Multilayer plasmonic photonic structures embedding photochromic molecules or optical gain molecules. Phys. E 2020, 120, 114081.

(34) Harrington, W. N.; Novoselova, M. V.; Bratashov, D. N.; Khlebtsov, B. N.; Gorin, D. A.; Galanzha, E. I.; Zharov, V. P. Photoswitchable Spasers with a Plasmonic Core and Photoswitchable Fluorescent Proteins. Sci. Rep. 2019, 9, 12439.

(35) Ono, M.; Hata, M.; Tsunekawa, M.; Nozaki, K.; Sumikura, H.; Chiba, H.; Notomi, M. Ultrafast and energy-efficient all-optical switching with graphene-loaded deepsubwavelength plasmonic waveguides. Nature Photonics 2020, 14, 37-43.

(36) Rekola, H. T.; Hakala, T. K.; Törmä, P. One-Dimensional Plasmonic Nanoparticle Chain Lasers. ACS Photonics 2018, 5, 1822-1826.

(37) Hakala, T. K.; Moilanen, A. J.; Väkeväinen, A. I.; Guo, R.; Martikainen, J.-P.; 
Daskalakis, K. S.; Rekola, H. T.; Julku, A.; Törmä, P. Bose-Einstein Condensation in a Plasmonic Lattice. Nat. Phys. 2018, 14, 739-744.

(38) De Giorgi, M.; Ramezani, M.; Todisco, F.; Halpin, A.; Caputo, D.; Fieramosca, A.; Gomez-Rivas, J.; Sanvitto, D. Interaction and Coherence of a Plasmon-Exciton Polariton Condensate. ACS Photonics 2018, 5, 3666-3672.

(39) Väkeväinen, A. I.; Moilanen, A. J.; Nečada, M.; Hakala, T. K.; Daskalakis, K. S.; Törmä, P. Sub-picosecond thermalization dynamics in condensation of strongly coupled lattice plasmons. Nat. Commun. 2020, 11, 3139.

(40) Martikainen, J.-P.; Moilanen, A. J.; Törmä, P. Coupled dipole approximation across the $\Gamma$-point in a finite-sized nanoparticle array. Philos. Trans. R. Soc., A 2017, 375, 20160316.

(41) Daskalakis, K. S.; Väkeväinen, A. I.; Martikainen, J.-P.; Hakala, T. K.; Törmä, P. Ultrafast Pulse Generation in an Organic Nanoparticle-Array Laser. Nano Lett. 2018, $18,2658-2665$.

(42) Vyshnevyy, A. A.; Fedyanin, D. Y. Lasing threshold of thresholdless and nonthresholdless metal-semiconductor nanolasers. Opt. Express 2018, 26, 33473-33483.

(43) Ruiz, C. C.; Hierrezuelo, J. M.; Molina-Bolivar, J. A. Analysis of the Photophysical Behavior and Rotational-Relaxation Dynamics of Coumarin 6 in Nonionic Micellar Environments: The Effect of Temperature. Molecules 2015, 20, 19343-19360.

(44) Laubereau, A.; Seilmeier, A.; Kaiser, W. A new technique to measure ultrashort vibrational relaxation times in liquid systems. Chem. Phys. Lett. 1975, 36, 232-237.

(45) Murakami, H. Femtosecond time-resolved fluorescence spectra of a coumarin dye in glycerol. J. Mol. Liq. 2000, 89, 33-45. 
(46) Hoang, T. B.; Akselrod, G. M.; Yang, A.; Odom, T. W.; Mikkelsen, M. H. MillimeterScale Spatial Coherence from a Plasmon Laser. Nano Lett. 2017, 17, 6690-6695.

(47) Irie, M. Diarylethenes for Memories and Switches. Chem. Rev. 2000, 100, 1685-1716.

(48) Gonçalves, P. A. D.; Christensen, T.; Rivera, N.; Jauho, A.-P.; Mortensen, N. A.; Soljačić, M. Plasmon-emitter interactions at the nanoscale. Nat. Commun. 2020, 11, 366.

(49) Johnson, P. B.; Christy, R. W. Optical Constants of the Noble Metals. Phys. Rev. B 1972, 6, 4370-4379.

(50) Meier, M.; Wokaun, A. Enhanced fields on large metal particles: dynamic depolarization. Opt. Lett. 1983, 8, 581-583.

(51) Steshenko, S.; Capolino, F. In Theory and Phenomena of Metamaterials; Capolino, F., Ed.; CRC Press: Boca Raton, 2009; Chapter 8.

(52) Draine, B. T.; Flatau, P. J. Discrete-Dipole Approximation For Scattering Calculations. J. Opt. Soc. Am. A 1994, 11, 1491-1499. 


\section{For Table of Contents Use Only}

Manuscript title: All-Optical Emission Control and Lasing in Plasmonic Lattices

Authors: Jani M. Taskinen, Antti J. Moilanen, Heikki Rekola, Kim Kuntze, Arri Priimagi,

Päivi Törmä, and Tommi K. Hakala

Brief synopsis of the TOC-graphic: All-optical control of highly directional lasing is achieved by combining plasmonic nanoparticle lattices with an organic gain dye and photochromic molecules, which can be optically switched between two conformational states.

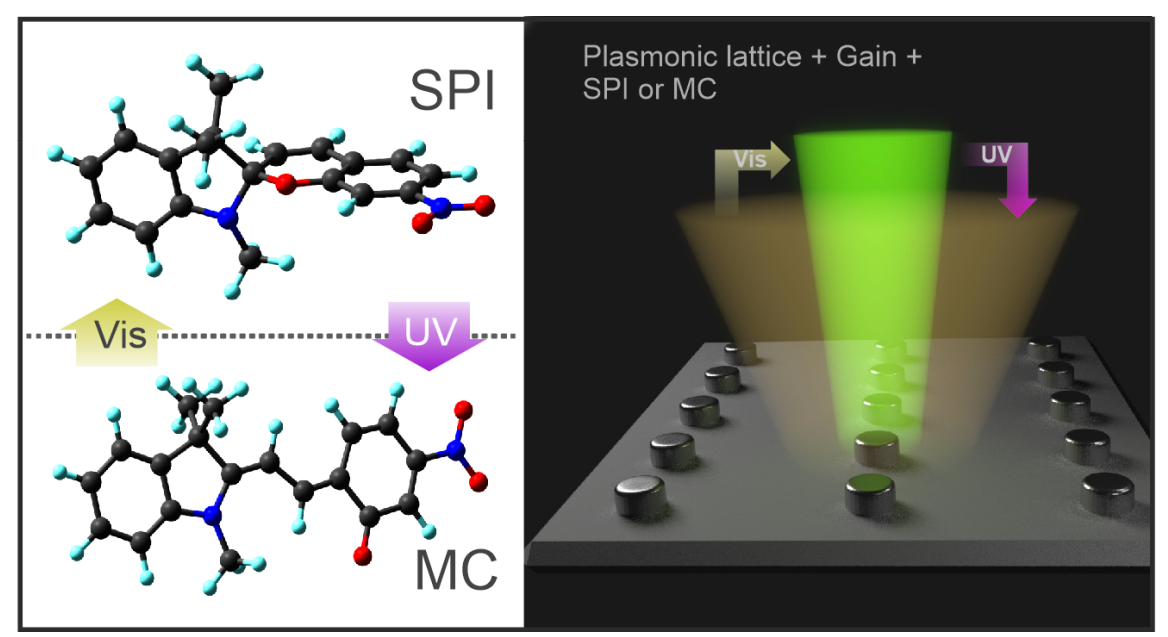

